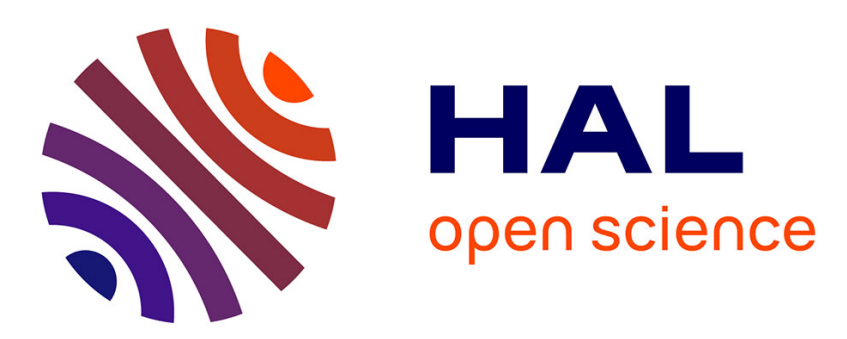

\title{
Extended Lie Brackets for Nonlinear Time-Delay Systems
}

Claudia Califano, Luis-Alejandro Marquez Martinez, Claude H. Moog

\section{To cite this version:}

Claudia Califano, Luis-Alejandro Marquez Martinez, Claude H. Moog. Extended Lie Brackets for Nonlinear Time-Delay Systems. IEEE Transactions on Automatic Control, 2011, 56 (9), pp.22132218. 10.1109/TAC.2011.2157405 . hal-00625401

\section{HAL Id: hal-00625401 https://hal.science/hal-00625401}

Submitted on 7 Oct 2011

HAL is a multi-disciplinary open access archive for the deposit and dissemination of scientific research documents, whether they are published or not. The documents may come from teaching and research institutions in France or abroad, or from public or private research centers.
L'archive ouverte pluridisciplinaire HAL, est destinée au dépôt et à la diffusion de documents scientifiques de niveau recherche, publiés ou non, émanant des établissements d'enseignement et de recherche français ou étrangers, des laboratoires publics ou privés. 


\section{Extended Lie Brackets for Nonlinear Time-Delay Systems}

Claudia Califano, Member, IEEE,

Luis Alejandro Marquez-Martinez, and

Claude H. Moog, Fellow, IEEE

\begin{abstract}
In this note the Extended Lie bracket operator is introduced for the analysis and control of nonlinear time-delay systems (NLTDS). This tool is used to characterize the integrability conditions of a given submodule. The obtained results have two fundamental outcomes. First, they define the necessary and sufficient conditions under which a given set of nonlinear one-forms in the $\boldsymbol{n}$-dimensional delayed variables $x(t), \cdots, x(t-s D)$, with $D$ constant but unknown, are integrable, thus generalizing the well known fundamental Frobenius Theorem to delay systems. Secondly, they set the basis for the extension to this context of the geometric approach used for delay-free systems. The effectiveness of the results is shown by solving the problem of the equivalence of a NLTDS to an accessible Linear Time-Delay System (LTDS) by bicausal change of coordinates.
\end{abstract}

Index Terms-Delay systems, geometric approach, linear equivalence, nonlinear continuous-time systems.

\section{INTRODUCTION}

Time-delay systems are recently gaining more and more attention due to their importance in several applications such as those concerning the delay in the signal transmission over communication networks. The literature on the topic is extensive but concerns mainly linear timedelay systems [1], [2], [8], [14], [19], [22], [25], [26] and does not make use of geometric tools which are instead largely used in the delay free-case both in the linear and nonlinear context, [10], [21], [27]. A first attempt to extend these tools to this class of infinite dimensional systems has been pursued in [24] and [23] with reference to the inputoutput linearization problem and the feedback linearization problem for a particular class of delay systems.

In this note we will consider nonlinear time-delay systems with commensurate constant delays on the state and input variables-see for example [6], [7], [12]—and we will introduce an Extended Lie bracket operation to deal with this class of systems. As shown in Section III, a major technical achievement is that this operation fully characterizes the integrability of a distribution defined on the extended state space. This tool is then used to derive necessary and sufficient integrability conditions of the given submodule. Let us note that a first attempt to deal with the integrability of one-forms was pursued in [17] where a necessary condition was derived and only some special cases were treated. The effectiveness of the proposed approach is shown by characterizing the necessary and sufficient conditions under which a NLTDS is equivalent to an accessible LTDS by bicausal change of coordinates, a largely studied problem in the delay free case, as proven by the extensive literature on the topic (e.g. [3], [11], [13], [15], [16],

Manuscript received February 17, 2010; revised August 18, 2010; accepted April 19, 2011. Date of publication May 23, 2011; date of current version September 08, 2011. Recommended by Associate Editor H. Ito.

C. Califano is with the Dip. di Inform. e Sistemistica "Antonio Ruberti," Università di Roma "La Sapienza," Rome 00185, Italy (e-mail: claudia.califano@uniroma1.it).

L. A. Marquez-Martinez is with the CICESE Research Center, Ensenada 22860, Mexico (e-mail: marquez@cicese.mx).

C. H. Moog is with the L'UNAM, IRCCyN, UMR C.N.R.S. Nantes 44321, France (e-mail: moog@ieee.org).

Color versions of one or more of the figures in this technical note are available online at http://ieeexplore.ieee.org.

Digital Object Identifier 10.1109/TAC.2011.2157405
[20]). With respect to [5], [24] we will consider a more general class of systems where there is no assumption on the delay of the input and we will study the effect of bicausal change of coordinates on the given system. For notational simplicity, and without loss of generality, we will consider as maximal delay the largest between the maximal delays on the state and input variables. Preliminary results can be found in [4].

The technical note is organized as follows. Some fundamental notions on time-delay systems are recalled and some notations are introduced in Section II. In Section III geometric tools for dealing with time-delay systems are introduced and discussed. In Section IV the proposed approach is used to solve the linear equivalence problem under bicausal change of coordinates.

\section{PRELIMINARIES AND NOTATIONS}

The following notations and definitions, borrowed from [9], [18], [28], will be used: $\mathcal{K}$ denotes the field of meromorphic functions of a finite number of elements in $\left\{x(t-i), u(t-i), \cdots, u^{(k)}(t-i), i, k \in\right.$ $I N\} ; d$ is the standard differential operator that maps elements from $\mathcal{K}$ to $\mathcal{E}=\operatorname{span}_{\mathcal{K}}\left\{d x(t-i), d u(t-i), \cdots, d u^{(k)}(t-i), i, k \in I N\right\}$ $\delta$ is the backward time-shift operator: for $a(\cdot), f(\cdot) \in \mathcal{K}$, $\delta(a(t) d f(t))=a(t-1) \delta d f(t)=a(t-1) d f(t-1)$. We will denote by deg. the polynomial degree in $\delta$ of its argument; $\mathbb{I R}[\delta]$ is the ring of polynomials in $\delta$ with coefficients in $\mathbb{R} ; \mathcal{K}(\delta]$ is the (left) ring of polynomials in $\delta$ with coefficients in $\mathcal{K}$. Every element of $\mathcal{K}(\delta]$ may be written as $\alpha(\delta]=\sum_{i=0}^{r_{\alpha}} \alpha_{i}(t) \delta^{i}$, with $\alpha_{i} \in \mathcal{K}$, and $r_{\alpha}=\operatorname{deg}(\alpha(\delta])$. Addition and multiplication on this ring, which is non-commutative but Euclidean [28], are defined by $\alpha(\delta]+\beta(\delta]=\sum_{i=0}^{\max \left\{r_{\alpha}, r_{\beta}\right\}}\left(\alpha_{i}(t)+\right.$ $\left.\beta_{i}(t)\right) \delta^{i}$ and $\alpha(\delta] \beta(\delta]=\sum_{i=0}^{r_{\alpha}} \sum_{j=0}^{r_{\beta}} \alpha_{i}(t) \beta_{j}(t-i) \delta^{i+j}$. Given $x(t) \in \mathbb{R}^{n}, \mathbf{x}_{[s]}^{T}=\left(x^{T}(t), \cdots x^{T}(t-s)\right)$ and $\left(\mathbf{x}_{[s]}(-p)\right)^{T}=$ $\left(x^{T}(t-p), \cdots x^{T}(t-s-p)\right)$, accordingly $\left.f\left(\mathbf{x}_{[l]}\right)\right|_{\mathbf{x}(-j)}:=$ $f(x(t-j), \cdots, x(t-j-l)) ; \mathbf{u}_{[s]}, \mathbf{u}_{[s]}(-p), \mathbf{z}_{[s]}$, and $\mathbf{z}_{[s]}(-p)$ are defined in a similar vein. When no confusion is possible the subindex will be omitted so that $\mathbf{x}$ will stand for $\mathbf{x}_{[s]}$ and $\mathbf{x}(-p)$ will stand for $\mathbf{x}_{[s]}(-p)$. Finally we will denote by $\mathbf{u}^{[j]}:=\left(\mathbf{u}, \dot{\mathbf{u}}, \cdots \mathbf{u}^{(j)}\right)$ for $j \geq 0$ while $\mathbf{u}^{[-1]}:=\emptyset$. Given $\tau^{T}(\mathbf{x})=\left[\tau_{1}(\mathbf{x}), \cdots, \tau_{n}(\mathbf{x})\right]$, we will denote by $\tau^{T}(\mathbf{x})(\partial / \partial x(t-j))=\sum_{i=1}^{n} \tau_{i}(\mathbf{x})\left(\partial / \partial x_{i}(t-j)\right)$ the submodule element which has component $\tau_{i}(\mathbf{x})$ in position $n j+i$. $\Delta=\operatorname{span}_{\mathcal{K}(\delta]}\left\{r_{1}(\mathbf{x}, \delta), \cdots, r_{j}(\mathbf{x}, \delta)\right\}$, is the right module spanned over $\mathcal{K}(\delta]$ by the column elements $r_{1}(\mathbf{x}, \delta), \cdots, r_{j}(\mathbf{x}, \delta) \in \mathcal{K}^{n}(\delta]$. Finally, let us recall that a polynomial matrix $A(\mathrm{x}, \delta) \in \mathcal{K}^{n \times n}(\delta]$ is called unimodular if it has a polynomial inverse. If $s=\operatorname{deg}(A)$ then $\operatorname{deg}\left(A^{-1}\right) \leq(n-1) s,[4]$.

In the following we will consider a nonlinear dynamics with integer delays $\Sigma$, represented as: ${ }^{1}$

$$
\Sigma: \dot{x}(t)=F\left(\mathrm{x}_{[s]}\right)+\sum_{j=0}^{s} G_{j}\left(\mathrm{x}_{[s]}\right) u(t-j)
$$

with $x \in \mathbb{R}^{n}, u \in \mathbb{R}$. We have that $\Sigma_{L}$, the variational or differential form representation of $\Sigma$, is given by

$$
\Sigma_{L}: d \dot{x}=f\left(\mathbf{x}_{[s]}, \mathbf{u}_{[s]}, \delta\right) d x+g\left(\mathbf{x}_{[s]}, \delta\right) d u
$$

with $g\left(\mathbf{x}_{[s]}, \delta\right)=\sum_{j=0}^{s} G_{j}\left(\mathbf{x}_{[s]}\right) \delta^{j}$ and $f\left(\mathbf{x}_{[s]}, \mathbf{u}_{[s]}, \delta\right)=$ $\sum_{i=0}^{s}\left(\left(\partial F\left(\mathbf{x}_{[s]}\right) / \partial x(t-i)\right)+\sum_{j=0}^{s} u(t-j)\left(\partial G_{j}\left(\mathbf{x}_{[s]}\right) / \partial x(t-\right.\right.$ i))) $\delta^{i}$.

${ }^{1}$ Note that considering integer delays is not restrictive since any commensurable delay system, where all the delays are multiples of a base constant delay $D$, can be put into this form by time scaling. 
In this context the following definition of bicausal change of coordinates [18] must be recalled.

Definition 1: Consider the dynamics $\Sigma$ with state coordinates $x$. $z=\phi\left(\mathbf{x}_{[\alpha]}\right), \phi \in \mathcal{K}^{n}$ is a bicausal change of coordinates for $\Sigma$ if there exist an integer $\ell \in N$ and a function $\phi^{-1}\left(\mathbf{z}_{[\ell]}\right) \in \mathcal{K}^{n}$ such that $x(t)=\phi^{-1}\left(\mathbf{z}_{[\ell]}\right)$.

Let $d z=T\left(\mathbf{x}_{[\gamma]}, \delta\right) d x$ be the differential form representation of the bicausal change of coordinates $z(t)=\phi\left(\mathbf{x}_{[\alpha]}\right)$ :

- $T\left(\mathbf{x}_{[\gamma]}, \delta\right)=\sum_{i=0}^{\alpha}\left(\partial \phi\left(\mathbf{x}_{[\alpha]}\right) / \partial x(t-i)\right) \delta^{i}=\sum_{i=0}^{\alpha} T^{i}\left(\mathbf{x}_{[\gamma]}\right) \delta^{i}$ is unimodular and $\gamma \leq \alpha$.

- The inverse $x=\phi^{-1}\left(\mathbf{z}_{[\ell]}\right)$ is characterized by $\ell \leq(n-1) \alpha$, and its differential representation is given by $d x=T^{-1}(\mathbf{z}, \delta) d z$, with $T^{-1}(\mathbf{z}, \delta)=\sum_{i=0}^{\ell}\left(\partial \phi^{-1}\left(\mathbf{z}_{[\ell]}\right) / \partial z(t-i)\right) \delta^{i}=$ $\left.T^{-1}(\mathbf{x}, \delta)\right|_{\mathbf{x}=\phi^{-1}\left(\mathbf{z}_{[\ell]}\right)}$.

- The differential form (2) is transformed into $d \dot{z}(t)=$ $\tilde{f}(\mathbf{z}, \mathbf{u}, \delta) d z+\tilde{g}(\mathbf{z}, \delta) d u$, with

$$
\begin{aligned}
\tilde{f}(\mathbf{z}, \mathbf{u}, \delta)=[ & (T(\mathbf{x}, \delta) f(\mathbf{x}, \mathbf{u}, \delta)+\dot{T}(\mathbf{x}, \delta)) \\
& \left.\times T^{-1}(\mathbf{x}, \delta)\right]_{\mid \mathbf{x}=\phi^{-1}(\mathbf{z})} \\
\tilde{g}(\mathbf{z}, \delta)=( & T(\mathbf{x}, \delta) g(\mathbf{x}, \delta))_{\mid \mathbf{x}=\phi^{-1}(\mathbf{z})} .
\end{aligned}
$$

\section{GeOMetry OF Time-Delay Systems}

The Extended Lie bracket is now introduced. It is shown how, through its application, it is possible to answer to some basic questions in control theory which were open problems up to now, such as: when a given set of one-forms depending on the state variable $x(t)$ and its delays $x(t-i), i \in[1, s]$, is integrable; when a given square matrix $T(\mathbf{x}, \delta)$ is the differential representation of a bicausal change of coordinates.

Definition 2: Let $r_{\beta}(\mathbf{x}, \mathbf{u}, \delta)=\sum_{j=0}^{s} r_{\beta}^{j}(\mathbf{x}, \mathbf{u}) \delta^{j}$, with $\beta=1,2$ and set $r_{\beta}^{j}(\cdot, \mathbf{u})=0$, for $j>s$. Then, $\forall k, l \geq 0$, the Extended Lie bracket $\left[r_{1}^{k}(\cdot, \mathbf{u}), r_{2}^{l}(\cdot, \mathbf{u})\right]_{E i}$, on $\mathbb{R}^{(i+1) n}, i \geq 0$, is defined as

$$
\begin{aligned}
& {\left[r_{1}^{k}(\cdot, \mathbf{u}), r_{2}^{l}(\cdot, \mathbf{u})\right]_{E i}} \\
& =\sum_{j=0}^{\min (k, l, i)}\left(\left[r_{1}^{k-j}(\cdot, \mathbf{u}), r_{2}^{l-j}(\cdot, \mathbf{u})\right]_{E 0}\right)_{\mid(\mathbf{x}(-j), \mathbf{u}(-j))}^{T} \\
& \quad \times \frac{\partial}{\partial x(t-j)}
\end{aligned}
$$

with

$$
\begin{aligned}
{\left[r_{1}^{k}(\cdot, \mathbf{u}), r_{2}^{l}(\cdot, \mathbf{u})\right]_{E 0}=} & \sum_{i=0}^{k} \frac{\partial r_{2}^{l}(\mathbf{x}, \mathbf{u})}{\partial x(t-i)} r_{1}^{k-i}(\mathbf{x}(-i), \mathbf{u}(-i))+ \\
& -\sum_{i=0}^{l} \frac{\partial r_{1}^{k}(\mathbf{x}, \mathbf{u})}{\partial x(t-i)} r_{2}^{l-i}(\mathbf{x}(-i), \mathbf{u}(-i)) .
\end{aligned}
$$

By definition the Extended Lie Bracket is antisymmetric.

Remark: The Extended Lie bracket definition is related to the infinite dimensional system

$$
\begin{aligned}
\dot{x}(t) & =F\left(\mathrm{x}_{[s]}\right)+\sum_{j=0}^{s} G_{j}\left(\mathrm{x}_{[s]}\right) u(t-j) \\
\dot{x}(t-1) & =F\left(\mathrm{x}_{[s]}(-1)\right)+\sum_{j=0}^{s} G_{j}\left(\mathrm{x}_{[s]}(-1)\right) u(t-j-1)
\end{aligned}
$$

naturally associated to the delay system (1). As a consequence if $r_{i}(\mathbf{x}, \delta), i \in[1, j]$ is associated to the dynamics $\dot{x}(t)=$
$F\left(\mathbf{x}_{[s]}\right)+\sum_{j=0}^{s} G_{j}\left(\mathbf{x}_{[s]}\right) u(t-j)$, then to $\dot{x}(t-1)=F\left(\mathbf{x}_{[s]}(-1)\right)+$ $\sum_{j=0}^{s} G_{j}\left(\mathbf{x}_{[s]}(-1)\right) u(t-j-1)$ it will be associated $\delta r_{i}(\mathbf{x}, \delta)$ and so on, that is

$$
\left\{\begin{array}{ccccccc}
r^{0} & r^{1} & \cdots & r^{s} & 0 & \cdots & \\
0 & r^{0}(-1) & \cdots & r^{s-1}(-1) & r^{s}(-1) & 0 & \ddots \\
\vdots & \ddots & \ddots & \vdots & \vdots & \ddots & \cdots \\
0 & \cdots & 0 & r^{0}(-s) & r^{1}(-s) & \cdots & \ddots \\
\vdots & \vdots & \vdots & \vdots & \vdots & \vdots & \ldots
\end{array}\right\}
$$

with $r^{i}(-p)=\left(r_{1}^{i}(\mathbf{x}(-p)), \cdots, r_{j}^{i}(\mathbf{x}(-p))\right)$. Despite the infinite dimensionality of the state space $x_{e}=$ $\left(x^{T}(t), x^{T}(t-1), x^{T}(t-2), \cdots\right)^{T}$ these elements are finite-dimensional so that we can thus consider the Lie bracket $\left[R_{1}^{j_{1}}, R_{2}^{j_{2}}\right]$ with $R_{l}^{j_{l}}=\sum_{i=0}^{j_{l}}\left(r_{l}^{j_{l}-i}(\mathbf{x}(-i))\right)^{T}(\partial / \partial x(t-i)), l=1,2$. Denoting by $j=\min \left(j_{1}, j_{2}\right),\left[R_{1}^{j_{1}}, R_{2}^{j_{2}}\right]=\left[r_{1}^{j_{1}}(\cdot), r_{2}^{j_{2}}(\cdot)\right]_{E j}$. The Extended Lie bracket (3) is thus a projection on the $(i+1) n$-dimensional state space.

Remark: The delayed state bracket introduced in [24] does not yield a criterion for the integrability of one-forms intrinsically depending on the delayed state variables as no decisive difference is made between the non integrable one-form $\omega_{1}(\mathbf{x}, \delta) d x=d x_{1}-x_{2}(t-1) d x_{2}$ and the exact one-form $\omega_{2}(\mathbf{x}, \delta) d x=d x_{1}-x_{2}(t-1) \delta d x_{2}$. The use of the Extended Lie bracket allows to make the difference as shown in the Examples 1 and 2.

To deal with the integrability of one-forms, we need now to introduce the following definition of integrable submodule. Let $r_{l}(\mathbf{x}, \delta)=\sum_{i=0}^{s} r_{l}^{i}(\mathbf{x}) \delta^{i}, l \in[1, j]$ and set $\Delta=\operatorname{span}_{\mathcal{K}(\delta]}\left\{r_{1}(\mathbf{x}, \delta), \cdots, r_{j}(\mathbf{x}, \delta)\right\}$, and $\mathbf{x}^{0}=\left(x^{0}(t)^{T}, \cdots, x^{0}(t-\gamma)^{T}\right)^{T}$.

Definition 3: The submodule $\Delta$ is nonsingular locally around $\mathrm{x}^{0}$ if $\operatorname{rank}(\Delta(\mathbf{x}))=j, \forall \mathbf{x} \in \mathcal{U}_{0}$ an open and dense subset of $\mathbf{x}^{0}$.

Definition 4: The submodule $\Delta$ nonsingular locally around $\mathbf{x}^{0}$, is integrable if there exist $n-j$ independent functions $\lambda_{l}\left(\mathbf{x}_{[\gamma]}\right), l \in$ $[1, n-j]$ such that $\operatorname{rank}(\partial \lambda(\mathbf{x}) / \partial x(t))=n-j$ and

$$
\sum_{p=0}^{\gamma} \frac{\partial \lambda_{l}(\mathbf{x})}{\partial x(t-p)} \delta^{p} \sum_{k=0}^{s} r_{i}^{k}(\mathbf{x}) \delta^{k}=0, \quad \forall l \in[1, n-j], \forall i \in[1, j] .
$$

Definition 5: Consider the bicausal change of coordinates $z=\phi\left(\mathbf{x}_{[\alpha]}\right)$, with $d z=T(\mathbf{x}, \delta) d x$. In the new coordinates the submodule element $r(\mathbf{x}, \mathbf{u}, \delta)$ is transformed as

$$
\tilde{r}(\mathbf{z}, \mathbf{u}, \delta)=[T(\mathbf{x}, \delta) r(\mathbf{x}, \mathbf{u}, \delta)]_{\mid \mathbf{x}=\phi^{-1}(\mathbf{z})} .
$$

From (7), setting $T^{j}=0$ for $j>\alpha=\operatorname{deg}(T(\mathbf{x}, \delta))$ and $r^{j}=0$ for $j>\operatorname{deg}(r(\mathbf{x}, \delta))$ one has

$$
\tilde{r}^{l}(\mathbf{z}, \mathbf{u})=\sum_{p=0}^{l}\left(T^{p}(\mathbf{x}) r^{l-p}(\mathbf{x}(-p), \mathbf{u}(-p))\right)_{\mid \mathbf{x}=\phi-1(\mathbf{z})} .
$$

Accordingly, from (8), the following result holds true.

Lemma 1: Let $r_{\beta}(\mathbf{x}, \mathbf{u}, \delta)=\sum_{j=0}^{s} r_{\beta}^{j}(\mathbf{x}, \mathbf{u}) \delta^{j}, \beta=1,2$. Under the bicausal change of coordinates $z=\phi\left(\mathbf{x}_{[\alpha]}\right)$, with $d z=T(\mathbf{x}, \delta) d x=\sum_{j=0}^{\alpha} T^{j}(\mathbf{x}) \delta^{j} d x$ one has, for $k \geq l, 0 \leq i \leq l$

$\left[\tilde{r}_{1}^{k}(\mathbf{z}, \mathbf{u}), \tilde{r}_{2}^{l}(\mathbf{z}, \mathbf{u})\right]_{E i}=\left(\Gamma^{l, i}(\mathbf{x})\left[r_{1}^{k}(\mathbf{x}, \mathbf{u}), r_{2}^{l}(\mathbf{x}, \mathbf{u})\right]_{E l}\right)_{\mid \mathbf{x}=\phi-1(\mathbf{z})}$

where, setting $T^{j}=0$ for $j>\alpha$

$$
\Gamma^{l, i}(\mathbf{x})=\left(\begin{array}{ccccc}
T^{0}(\mathbf{x}) & \cdots & \cdots & \cdots & T^{l}(\mathbf{x}) \\
0 & \ddots & & & \vdots \\
0 & 0 & T^{0}(\mathbf{x}(-i)) & \cdots & T^{l-i}(\mathbf{x}(-i))
\end{array}\right) .
$$


The proof, omitted for space reasons, can be carried out by substituting in $\left[\tilde{r}_{1}^{k}(\mathbf{z}, \mathbf{u}), \tilde{r}_{2}^{l}(\mathbf{z}, \mathbf{u})\right]_{E 0}$ the relations given by (8) and by recalling that $\partial\left(T^{p}(\mathbf{x})\right)^{T} / \partial x(t-i)=\left(\partial\left(T^{i}(\mathbf{x})\right)^{T} / \partial x(t-p)\right)^{T}$.

\section{A. Integrability Conditions of One-Forms}

We will now address the problem of defining the necessary and sufficient conditions under which some given one-forms are integrable. The obtained results are based on the consideration that though when dealing with delay systems one ends up on the infinite dimensional system (5), the elements that one considers are characterized by a finite number of components. This is enlightened in next Lemma which displays the properties of the Extended Lie bracket operator.

Lemma 2: Let $\Delta=\operatorname{span}_{\mathcal{K}(\delta]}\left\{r_{1}(\mathbf{x}, \delta), \cdots, r_{j}(\mathbf{x}, \delta)\right\}$ with $r_{i}(\mathbf{x}, \delta)=\sum_{l=0}^{s} r_{i}^{l}\left(\mathbf{x}_{[\alpha]}\right) \delta^{l}, s=\max \left\{\alpha, \operatorname{deg}\left(r_{i}(\mathbf{x}, \delta)\right), i=1,2\right\}$. Then for any couple of integers $l \leq k$, and for any integer $\gamma, \bar{\gamma}, p \geq 0$ such that $\gamma \geq l$ and $\bar{\gamma} \geq l+p s$, the following properties hold true:

$$
\begin{aligned}
{\left[r_{i}^{l}, r_{j}^{k}\right]_{E \gamma} \equiv } & {\left[r_{i}^{l}, r_{j}^{k}\right]_{E l} } \\
= & \sum_{\beta=0}^{l}\left(\left[r_{i}^{l-\beta}(\cdot), r_{t}^{k-\beta}(\cdot)\right]_{E 0}\right)_{\mid(\mathbf{x}(-\beta))}^{T} \\
& \times \frac{\partial}{\partial x(t-\beta)} \\
{\left[r_{i}^{l+p s}, r_{j}^{k+p s}\right]_{E \bar{\gamma}} \equiv } & {\left[r_{i}^{l+p s}, r_{j}^{k+p s}\right]_{E, l+p s} } \\
= & \sum_{\beta=0}^{l}\left(\left[r_{i}^{l-\beta}(\cdot), r_{j}^{k-\beta}(\cdot)\right]_{E 0}\right)_{\mid(\mathbf{x}(-\beta-p s))}^{T} \\
& \times \frac{\partial}{\partial x(t-\beta-p s)}, l \geq s \\
\equiv & {\left[r_{i}^{l}, r_{j}^{k}\right]_{E l}=0, \quad k-l>2 s }
\end{aligned}
$$

Sketch of Proof: The proof of $(9) \div(11)$ is immediate if one considers the elements $R_{j}^{k}(\mathbf{x})=\sum_{i=0}^{k} r_{j}^{k-i}(\mathbf{x}(-i))(\partial / \partial x(t-i))$ defined on (6). In fact on the extended state space $\left(\partial R_{i}^{l} / \partial x_{e}\right) R_{j}^{k}-$ $\left(\partial R_{j}^{k} / \partial x_{e}\right) R_{i}^{l}=0$ whenever $|k-l|>2 s$, while the others yield the same equations, only time and coordinates -shifted.

Next theorem, whose proof is reported in the Appendix, enlightens the conditions under which $n$ one-forms are exact and define a bicausal change of coordinates. The conditions are given on the corresponding submodule elements. It is shown that the nilpotency condition of a given Lie Algebra which is the key point in the case of nonlinear systems without delays is transformed into a nilpotency condition on the given submodule which takes into account not only the state variable $x(t)$ but also the delayed variables. The bound on the delay is defined by the state dimension and maximal delay.

Theorem 1: Let $P_{n}(\mathbf{x}, \delta)=\left[r_{1}(\mathbf{x}, \delta), \cdots, r_{n}(\mathbf{x}, \delta)\right] \in \mathcal{K}^{n \times n}(\delta]$ be a full rank matrix, with $r_{i}(\mathbf{x}, \delta)=\sum_{j=0}^{s} r_{i}^{j}\left(\mathbf{x}_{[\alpha]}\right) \delta^{j}$, $s=\max \left\{\alpha, \operatorname{deg}\left(r_{i}(\mathbf{x}, \delta)\right), i \in[1, n]\right\}$, which can be factorized as $P_{n}(\mathbf{x}, \delta)=T(\mathbf{x}, \delta) Q(\delta)$, with $T(\mathbf{x}, \delta)$ unimodular. Then $T^{-1}(\mathbf{x}, \delta)$ defines a bicausal change of coordinates iff

$$
\left[r_{j}^{i}(\mathbf{x}), r_{l}^{k}(\mathbf{x})\right]_{E, 2 s}=0 \forall l, j \in[1, n] \text { and } \forall i \leq k \in[0,2 s]
$$

or equivalently

$$
\left[r_{j}^{i}(\mathbf{x}), r_{l}^{k}(\mathbf{x})\right]_{E 0}=0 \forall l, j \in[1, n] \text { and } \forall i \leq k \in[0,2 s] .
$$

Consider now $P_{j}(\mathbf{x}, \delta)=\left[r_{1}(\mathbf{x}, \delta), \cdots, r_{j}(\mathbf{x}, \delta)\right]=$ $\sum_{l=0}^{s} P_{j l}(\mathbf{x}) \delta^{l}$ with $P_{j 0}(\mathbf{x})$ of rank $j$ and $r_{k}(\mathbf{x}, \delta)=\sum_{l=0}^{s} r_{k}^{s}(\mathbf{x}) \delta^{s}$, $k \in[1, j]$. Consider the distributions $\Delta_{i}$ and $\Delta_{i}^{\prime}, i \geq 0$ defined on $\mathbb{R}^{(i+1) n}$ and with vector fields parameterized by

$$
\begin{gathered}
x(t-i-1), \cdots, x(t-i-s) \\
\Delta_{i}=\operatorname{span}_{\mathcal{K}}\left\{\sum_{l=0}^{\gamma}\left(r_{k}^{\gamma-l}(\mathbf{x}(-l))\right)^{T} \frac{\partial}{\partial x(t-l)}, \begin{array}{l}
k \in[1, j] \\
\gamma \in[0, i]
\end{array}\right\}, \\
\Delta_{i}^{\prime}=\operatorname{span}_{\mathcal{K}}\left\{\begin{array}{c}
\sum_{l=0}^{\min (\gamma, i)}\left(r_{k}^{\gamma-l}(\mathbf{x}(-l))\right)^{T} \frac{\partial}{\partial x(t-l)}, \\
k \in[1, j] \\
\gamma \in[0, i+s]
\end{array}\right\} .
\end{gathered}
$$

By construction $\Delta_{i} \subseteq \Delta_{i}^{\prime}$. Let $\rho_{i}=\operatorname{rank}\left(\Delta_{i}^{\prime}\right)$ locally around $\mathrm{x}^{0}$, then $\Delta_{i}^{\prime}=\operatorname{span}\left\{\tau_{l}(\mathbf{x}), l \in\left[1, \rho_{i}\right]\right\} \subset \mathbb{R}^{(i+1) n}$ while its elements depend on the variables $\mathbf{x}_{[i+s]}$. Let us thus consider the series development of $\tau_{l}$ with respect to the parameters $\mathbf{x}(-i-1)$ locally around $\mathbf{x}^{0}(-i-1)$ which without loss of generality can be assumed to be the origin, that is

$$
\begin{aligned}
\tau_{l}(\mathbf{x})= & \tau_{l 0}\left(\mathbf{x}_{[i]}\right)+\sum_{j=1}^{s} \sum_{\alpha=1}^{n} \alpha_{j} \tau_{l 1}\left(\mathbf{x}_{[i]}\right) x_{\alpha}(-i-j) \\
& +\frac{1}{2} \sum_{\alpha, \beta=1}^{n} \sum_{j, k=1}^{s} \alpha_{j} \beta_{k} \tau_{l 2}\left(\mathbf{x}_{[i]}\right) x_{\alpha}(-i-j) x_{\beta}(-i-k)+\cdots
\end{aligned}
$$

and consider the possibly infinite set of distributions

$$
\begin{aligned}
& \Delta_{i 0}^{\prime}=\operatorname{span}\left\{\tau_{l 0}, l \in[1, k]\right\} \\
& \Delta_{i 1}^{\prime}=\operatorname{span}\left\{\alpha_{j} \tau_{l 1}, l \in[1, k], j \in[1, s], \alpha \in[1, n]\right\}
\end{aligned}
$$

Set $\rho_{i 0}=\operatorname{rank} \overline{\left(\sum_{k \geq 0} \Delta_{i k}^{\prime}\right)}$. We can now state the main result concerning integrability.

Theorem 2: Let $\Delta=\operatorname{span}_{\mathcal{K}(\delta]}\left\{r_{1}(\mathbf{x}, \delta), \cdots, r_{j}(\mathbf{x}, \delta)\right\}$ with $r_{i}(\mathbf{x}, \delta)=\sum_{l=0}^{s} r_{i}^{l}\left(\mathbf{x}_{[\alpha]}\right) \delta^{l}, s=\max \left\{\operatorname{deg}\left(r_{i}(\mathbf{x}, \delta), i \in[1, j], \alpha\right\}\right.$ and such that the matrices $P_{j}(\mathbf{x}, \delta)=\left(r_{1}(\mathbf{x}, \delta), \cdots, r_{j}(\mathbf{x}, \delta)\right)=$ $\sum_{l=0}^{s} P_{j l}(\mathbf{x}) \delta^{l}$ and $P_{j 0}(\mathbf{x})$ are of rank $j$. Let $\Delta_{i}^{\prime}$ and $\overline{\left(\sum_{k>0} \Delta_{i k}^{\prime}\right)}$ be the associated set of distributions defined, respectively, by (13) and (14) which are assumed to be locally non singular on $\mathbf{x}^{0}=\left(x^{0}(t)^{T}, \cdots x^{0}(t-i)^{T}\right)^{T}$ with $\rho_{i}=\operatorname{rank} \Delta_{i}^{\prime}$ and $\rho_{i 0}=\operatorname{rank} \overline{\left(\sum_{k>0} \Delta_{i k}^{\prime}\right)}$ (with $\rho_{-1}=\rho_{-1,0}=0$ ). Then $\Delta$ is integrable iff there exists an index $\gamma$ such that:

a) $\forall l, k \in[1, j]$ and $t \leq p \leq i+s,\left[r_{l}^{t}(\cdot), r_{k}^{p}(\cdot)\right]_{E i} \in \Delta_{i}^{\prime}, i \in$ $[0, \gamma]$

b) $\rho_{\gamma}-\rho_{\gamma-1}=j$;

c) $\rho_{\gamma 0}-\rho_{\gamma-1,0}=j$.

Proof: Necessity: Assume that there exist $n-j$ independent functions $\lambda_{i}(\mathbf{x}), i \in[1, n-j]$ with $\operatorname{rank}(\partial \lambda / \partial x(t))=n-j$ which are in the kernel of $P_{j}(\mathbf{x}, \delta)$. In the set of all possible independent solutions, consider the one characterized by the minimum delay. Denote by $\gamma$ the maximum delay in the functions $\lambda_{i}(\mathbf{x})$, then $d \lambda_{i}(\mathbf{x})=$ $\sum_{k=0}^{\gamma} \omega_{i}^{k}(\mathbf{x}) \delta^{k} d x$ satisfies

$$
\sum_{k=0}^{\gamma} \omega_{i}^{k}(\mathbf{x}) \delta^{k} \sum_{l=0}^{s} P_{j l}(\mathbf{x}) \delta^{l}=0
$$

It is easily seen that for any index $\beta \geq 0,\left[r_{l}^{t}(\cdot), r_{k}^{p}(\cdot)\right]_{E \beta} \in \Delta_{\beta}$, $\forall t \leq p \leq \beta$. As a consequence $\left[r_{l}^{t}(\cdot), r_{k}^{p}(\cdot)\right]_{E i} \in \Delta_{i}^{\prime}, \forall t \leq p \leq i+s$ which proves a).

Let $\gamma$ be the maximum delay in the functions $\lambda_{i}(\mathbf{x})$. If $\rho_{\gamma-1}=n \gamma-j_{\gamma-1}$, then there exist $j_{\gamma-1}$ independent one-forms $\bar{\omega}(\mathbf{x}, \delta) d x=\sum_{k=0}^{\gamma-1} \bar{\omega}^{k}(\mathbf{x}) d x(t-k)$ (not necessarily exact) such that $\left[\bar{\omega}^{0}(\mathbf{x}), \cdots, \bar{\omega}^{\gamma-1}(\mathbf{x})\right] \Delta_{\gamma-1}^{\prime}=0$. As a consequence

$$
\left(\begin{array}{cccc}
\omega^{0}(\mathbf{x}) & \omega^{1}(\mathbf{x}) & \cdots & \omega^{\gamma}(\mathbf{x}) \\
0 & \bar{\omega}^{0}(\mathbf{x}(-1)) & \cdots & \bar{\omega}^{\gamma-1}(\mathbf{x}(-1))
\end{array}\right) \Delta_{\gamma}^{\prime}=0
$$


with $\operatorname{rank}\left[\omega^{0}(\mathbf{x}), \omega^{1}(\mathbf{x}), \cdots, \omega^{\gamma}(\mathbf{x})\right]=n-j$, which implies that $\rho_{\gamma}=n(\gamma+1)-(n-j)-j_{\gamma-1}$, and thus b) must hold.

Let us finally consider the distributions $\overline{\left(\sum_{k>0} \Delta_{\gamma-1, k}^{\prime}\right)}$ and $\overline{\left(\sum_{k \geq 0} \Delta_{\gamma, k}^{\prime}\right)}$, and let $\rho_{\gamma-1,0}=n \gamma-k_{1}$, and $\rho_{\gamma, 0}=n(\gamma+1)-k_{2}$, $k_{1}, k_{2} \geq 0$. Then there exist $k_{1} \geq 0$ exact and independent one-forms $d \tilde{\lambda}_{i}\left(\mathbf{x}_{[\gamma-1]}\right)=\sum_{k=0}^{\gamma-1} \tilde{\omega}_{i}^{k}(\mathbf{x}) d x(t-k)$ such that

$$
\left[\tilde{\omega}^{0}(\mathbf{x}), \cdots, \tilde{\omega}^{\gamma-1}(\mathbf{x})\right] \overline{\left(\sum_{k \geq 0} \Delta_{\gamma-1, k}^{\prime}\right)}=0 .
$$

On the other hand the $n-j$ functions $\lambda_{i}(x(t), \cdots, x(t-\gamma))$ being independent on $x(t-k)$ for $k>\gamma$, implies that $d \lambda_{i}(x(t), \cdots, x(t-$ $\gamma))=\sum_{k=0}^{\gamma} \tilde{\omega}_{i}^{k}(\mathbf{x}) d x(t-k)$ satisfies

$$
\left[\omega^{0}(\mathbf{x}), \cdots, \omega^{\gamma}(\mathbf{x})\right] \overline{\left(\sum_{k \geq 0} \Delta_{\gamma, k}^{\prime}\right)}=0 .
$$

Since also $\left[0, \tilde{\omega}^{0}(\mathbf{x}(-1)), \cdots, \tilde{\omega}^{\gamma-1}(\mathbf{x}(-1))\right] \overline{\left(\sum_{k>0} \Delta_{\gamma, k}^{\prime}\right)}=0$ then $k_{2}=k_{1}+n-j$ and c) follows.

Sufficiency: Assume that conditions a) $\div$ c) are satisfied. Then by assumption due to a) and b) there exists an index $\gamma$ such that $\rho_{\gamma}-$ $\rho_{\gamma-1}=(\gamma+1) n-k_{1}-k_{2}-\left(n \gamma-k_{1}\right)=j$, that is, there exist $k_{2}=n-j$ independent one-forms $\sum_{k=0}^{\gamma} \omega_{i}^{k}(\mathbf{x}) d x(t-k)$ such that

$$
\left(\omega^{0}(\mathbf{x}) \quad \cdots \quad \omega^{\gamma}(\mathbf{x})\right) \bar{\Delta}_{\gamma}^{\prime}=0
$$

with $\operatorname{rank}\left(\omega^{0}(\mathbf{x})\right)=n-j$ locally around $\mathbf{x}^{0}$. Finally according to $\left.\mathrm{c}\right)$ the one-forms $\omega_{i}(\mathbf{x}, \delta) d x$ can be chosen to be exact, that is, there exist $n-j$ functions $\lambda_{i}(\mathbf{x})$ such that

$$
\left[\frac{\partial \lambda_{i}(\mathbf{x})}{\partial x(t)}, \cdots, \frac{\partial \lambda_{i}(\mathbf{x})}{\partial x(t-\gamma)}\right] \Delta_{\gamma}^{\prime}=0
$$

with $\operatorname{rank}(\partial \lambda(\mathbf{x}) / \partial x(t))=n-j$, which ends the proof.

Example 1: Let $\Delta=\operatorname{span}_{\mathcal{K}}\left\{x_{2}(t-1)\left(\partial / \partial x_{1}(t)\right)+\left(\partial / \partial x_{2}(t)\right)\right\}$. From Theorem 2 it follows that $\Delta$ is not integrable. In fact

$$
\Delta_{0}=\Delta_{0}^{\prime}=\operatorname{span}_{\mathcal{K}}\left\{x_{2}(t-1) \frac{\partial}{\partial x_{1}(t)}+\frac{\partial}{\partial x_{2}(t)}\right\}
$$

which satisfies $\left[r_{1}^{0}, r_{1}^{0}\right]_{E 0}=0 \in \Delta_{0}$, while

$$
\Delta_{1}=\Delta_{1}^{\prime}=\Delta_{0}+\operatorname{span}_{\mathcal{K}}\left\{x_{2}(t-2) \frac{\partial}{\partial x_{1}(t-1)}+\frac{\partial}{\partial x_{2}(t-1)}\right\}
$$

for which $\left[r_{1}^{0}, r_{1}^{1}\right]_{E 1}=-\left(\partial / \partial x_{1}(t)\right) \notin \Delta_{1}^{\prime}$, thus proving that the one-form $\omega_{1}(\mathbf{x}, \delta) d x=d x_{1}-x_{2}(t-1) d x_{2}$ which lies in the left kernel of $\Delta$ is not integrable.

Example 2: Consider the submodule $\Delta=\operatorname{span}_{\mathcal{K}(\delta]}\left\{x_{2}(t-\right.$ 1) $\left.x_{1}(t-1) \delta\left(\partial / \partial x_{1}(t)\right)+x_{1}(t)\left(\partial / \partial x_{2}(t)\right)\right\}$. According to Theorem 2 , to check if there exists a one-form which lies in the left kernel of $\Delta$, we must consider

$$
\begin{aligned}
& \Delta_{0}^{\prime}=\operatorname{span}_{\mathcal{K}}\left\{x_{1}(t) \frac{\partial}{\partial x_{2}(t)}, x_{2}(t-1) x_{1}(t-1) \frac{\partial}{\partial x_{1}(t)}\right\}, \\
& \Delta_{1}^{\prime}=\operatorname{span}_{\mathcal{K}}\left\{x_{1}(t) \frac{\partial}{\partial x_{2}(t)}, x_{2}(t-2) x_{1}(t-2) \frac{\partial}{\partial x_{1}(t-1)}\right\} \\
&+\operatorname{span}_{\mathcal{K}}\left\{x_{1}(t-1) x_{2}(t-1) \frac{\partial}{\partial x_{1}(t)}\right. \\
&\left.+x_{1}(t-1) \frac{\partial}{\partial x_{2}(t-1)}\right\},
\end{aligned}
$$

since $\rho_{0}=\rho_{00}=\operatorname{rank}\left(\Delta_{0}^{\prime}\right)=2$ locally around $x^{0} \neq 0$, then condition a) of Theorem 2 holds true for $\Delta_{0}^{\prime}$. As for $\Delta_{1}^{\prime}$, it is readily seen that it is independent of $x(t-i) i \geq 2$ and is involutive, so that condition a) is satisfied for $\Delta_{1}^{\prime}$; moreover $\rho_{1}=\rho_{1,0}=\operatorname{rank}\left(\Delta_{1}^{\prime}\right)=3$ so that also condition b) and c) are satisfied being $\rho_{1}-\rho_{0}=\rho_{10}-\rho_{00}=1$. Thus there exists one integrable one-form in the left kernel of $\Delta$ given by $\omega_{2}(\mathbf{x}, \delta) d x=d x_{1}-x_{2}(t-1) \delta d x_{2}=x_{1}(t)-(1 / 2)\left[x_{2}(t-1)\right]^{2}$.

\section{ApPlication to LineAR EQUiVALENCE of TDS}

It is now shown how the results derived in Section III can be efficiently used to solve the linear equivalence problem under bicausal change of coordinates.

Problem Statement (The Linear Equivalence Problem): Given system (1), find, if possible, a bicausal change of coordinates $z=\phi\left(\mathbf{x}_{[\alpha]}\right)$ such that

$$
\dot{z}=\sum_{j=1}^{l} A_{j} z(t-j)+\sum_{j=1}^{l} B_{j} u(t-j)
$$

with $A(\delta)=\sum_{j=1}^{l} A_{j} \delta^{j}, B(\delta)=\sum_{j=1}^{l} B_{j} \delta^{j}$ a weakly accessible pair.

Some preliminary definitions related to the accessibility properties of a NLTDS are in order.

\section{A. Accessibility Submodules}

Let $g_{1}\left(\mathbf{x}_{[s]}, \delta\right):=g\left(\mathbf{x}_{[s]}, \delta\right)$. The module generators $g_{k}\left(\mathbf{x}, \mathbf{u}^{[k-2]}, \delta\right), k \geq 2$ are recursively defined as

$g_{k}\left(\mathbf{x}, \mathbf{u}^{[k-2]}, \delta\right)=f(\mathbf{x}, \mathbf{u}, \delta) g_{k-1}\left(\mathbf{x}, \mathbf{u}^{[k-3]}, \delta\right)-\dot{g}_{k-1}\left(\mathbf{x}, \mathbf{u}^{[k-3]}, \delta\right)$.

Definition 6: The accessibility submodules $\mathcal{R}_{i}$ of $\Sigma$, are defined as $\mathcal{R}_{i}=\operatorname{span}_{\mathcal{K}(\delta]}\left\{g_{1}(\mathbf{x}, \delta) \cdots g_{i}\left(\mathbf{x}, \mathbf{u}^{[i-2]}, \delta\right)\right\}, i \geq 1$.

Remark: Note that for nonlinear time-delay systems, the left-kernel of $\mathcal{R}_{i}$ is the left-submodule $\mathcal{H}_{i+1}$, as shown in [28]. For nonlinear systems without delays

$$
\begin{aligned}
& g_{k}\left(x(t), u(t), \cdots, u^{(k-2)}(t)\right) \\
& \quad=(-1)^{k-1}\left(a d_{f}^{k-1} g+\left[g, a d_{f}^{k-2} g\right] u+\cdots\right)
\end{aligned}
$$

while for the linear time-varying and time-invariant cases, $\mathcal{R}_{n}$ reduces to the corresponding accessibility matrices $[B(t) A(t) B(t)-\dot{B}(t) \cdots]$, $\left[B A B \cdots A^{n-1} B\right]$ resp.

The following results hold true [4].

Proposition 1: If $g_{i+1}\left(\mathbf{x}, \mathbf{u}^{[i-1]}, \delta\right) \in \mathcal{R}_{i}$ then $\forall j \geq 1$, $g_{i+j}\left(\mathbf{x}, \mathbf{u}^{[i+j-2]}, \delta\right) \in \mathcal{R}_{i}$.

Proposition 2: Under the bicausal change of coordinates $z=\phi\left(\mathbf{x}_{[\alpha]}\right)$, with $d z=T(\mathbf{x}, \delta) d x$ the accessibility submodules elements $g_{j}(\cdot)$ are transformed, for $j \geq 1$, as

$$
\tilde{g}_{j}\left(\mathbf{z}, \mathbf{u}^{[j-2]}, \delta\right)=\left[T(\mathbf{x}, \delta) g_{j}\left(\mathbf{x}, \mathbf{u}^{[j-2]}, \delta\right)\right]_{\mathbf{x}=\phi-1(\mathbf{z})} .
$$

Corollary 1: Under a bicausal change of coordinates $z=\phi\left(\mathbf{x}_{[\alpha]}\right)$

$$
\begin{aligned}
\mathcal{R}_{i} & =\operatorname{span}_{\mathcal{K}(\delta]}\left\{g_{1}(\mathbf{x}, \delta) \cdots g_{i}\left(\mathbf{x}, \mathbf{u}^{[i-2]}, \delta\right)\right\} \equiv \tilde{\mathcal{R}}_{i} \\
& =\operatorname{span}_{\mathcal{K}(\delta]}\left\{\tilde{g}_{1}(\mathbf{z}, \delta) \cdots \tilde{g}_{i}\left(\mathbf{z}, \mathbf{u}^{[i-2]}, \delta\right)\right\} .
\end{aligned}
$$

\section{B. Linear Equivalence of Time-Delay Systems}

Theorem 3: System (1) is equivalent, under a bicausal change of coordinates, to a linear weakly accessible delay system if and only if there exist a unimodular matrix $T^{-1}(\mathbf{x}, \delta)$ and a full rank matrix $Q(\delta)$, such that 
a) the module generators $g_{i}$ 's do not depend on $\mathbf{u}^{[i-2]}$, that is, for $1 \leq i \leq n, g_{i}(\cdot):=g_{i}(\mathbf{x}, \delta)$

b) $R_{n}(\mathbf{x})=\left(g_{1}(\mathbf{x}, \delta), \cdots g_{n}(\mathbf{x}, \delta)\right)=T^{-1}(\mathbf{x}, \delta) Q(\delta)$;

c) $g_{n+1}(\cdot):=g_{n+1}(\mathbf{x}, \delta) \in \operatorname{span}_{\mathbb{R}(\delta]}\left\{g_{1}(\mathbf{x}, \delta), \cdots, g_{n}(\mathbf{x}, \delta)\right\}$;

d) denoting by $\bar{s}=\max \left(\operatorname{deg}\left(g_{l}\left(\mathbf{x}_{[\alpha]}, \delta\right)\right), l \in[1, n], \alpha\right)$, for $i, j \in$ $[1, n]$ and $r \leq k \in[0,2 \bar{s}]$

$$
\left[g_{i}^{r}(\mathbf{x}), g_{j}^{k}(\mathbf{x})\right]_{E, 2 \bar{s}}=0
$$

or equivalently

$$
\left[g_{i}^{r}(\mathbf{x}), g_{j}^{k}(\mathbf{x})\right]_{E 0}=0 .
$$

The proof, omitted for space reasons, can be carried out as in [4], by first showing that the conditions are satisfied by a weakly accessible LTDS and are invariant under bicausal change of coordinates, and then by showing that, if the conditions are satisfied, according to Theorem 1 the matrix $T^{-1}(\mathbf{x}, \delta)$ defines a bicausal change of coordinates and that in these new coordinates the system is linear.

Corollary 2: System (1) is equivalent, under a bicausal change of coordinates, to a delay-free linear accessible system iff conditions a) and d) of Theorem 3 are satisfied, and

b') $R_{n}(\mathbf{x})=\left(g_{1}(\mathbf{x}, \delta), \cdots g_{n}(\mathbf{x}, \delta)\right)$ is unimodular

c') $g_{n+1}(\cdot):=g_{n+1}(\mathbf{x}, \delta) \in \operatorname{span}_{\mathbb{R}}\left\{g_{1}(\mathbf{x}, \delta), \cdots g_{n}(\mathbf{x}, \delta)\right\}$.

Example 3: Consider the dynamics

$$
\begin{aligned}
& \dot{x}_{1}(t)=x_{2}(t)-x_{2}(t-1)+2 x_{2}(t-1) \sum_{p=1}^{2} u(t-p) \\
& \dot{x}_{2}(t)=u(t)+u(t-1)
\end{aligned}
$$

for which $g_{1}(\cdot)=\left[2 x_{2}(t-1)(\delta+1) \delta\right]\left(\partial / \partial x_{1}(t)\right)+(\delta+$ 1) $\left(\partial / \partial x_{2}(t)\right), g_{2}(\cdot)=\left(1-\delta^{2}\right)\left(\partial / \partial x_{1}(t)\right)$ and $g_{3}(\cdot)=0$.

Condition a) of Theorem 3 is satisfied and the accessibility matrix $R_{n}(\mathbf{x})$ is given by

$$
\begin{aligned}
R_{n}(\mathbf{x}) & =\left(\begin{array}{cc}
2 x_{2}(t-1)(\delta+1) \delta & 1-\delta^{2} \\
\delta+1 & 0
\end{array}\right) \\
& =\left(\begin{array}{cc}
2 x_{2}(t-1) \delta & 1 \\
1 & 0
\end{array}\right)\left(\begin{array}{cc}
\delta+1 & 0 \\
0 & 1-\delta^{2}
\end{array}\right) \\
& =T^{-1}(\mathbf{x}, \delta) Q(\delta) .
\end{aligned}
$$

$R_{n}(\mathbf{x})$ is not unimodular but it is factorized in the product of an unimodular matrix $T^{-1}(\mathbf{x}, \delta)$ and a full rank matrix $Q(\delta)$, which shows that condition b) is verified. Condition c) is also satisfied so we must only check condition d) with $\bar{s}=2$. We can equivalently consider the Extended Lie brackets $\left[g_{l}^{i}, g_{k}^{j}\right]_{E 0}$ with $l, k \in[1,2]$ and $i \leq j \in[0,4]$ with $g_{1}^{0}=\left(\partial / \partial x_{2}(t)\right), g_{1}^{1}=2 x_{2}(t-1)\left(\partial / \partial x_{1}(t)\right)+\left(\partial / \partial x_{2}(t)\right)$, $g_{1}^{2}=2 x_{2}(t-1)\left(\partial / \partial x_{1}(t)\right), g_{1}^{3}=g_{1}^{4}=0, g_{2}^{0}=\left(\partial / \partial x_{1}(t)\right), g_{2}^{1}=0$, $g_{2}^{2}=-\left(\partial / \partial x_{1}(t)\right), g_{2}^{3}=g_{2}^{4}=0$. Since these Extended Lie brackets are zero, the matrix $T^{-1}(\mathbf{x}, \delta)$ defines the bicausal change of coordinates $d z=\left(d\left(x_{2}(t)\right), d\left(x_{1}(t)-x_{2}^{2}(t-1)\right)\right)^{T}$ and yields

$$
\dot{z}_{1}(t)=u(t)+u(t-1), \quad \dot{z}_{2}(t)=z_{1}(t)+z_{1}(t-1) .
$$

\section{CONCLUSION}

In this note a geometric approach for the analysis of the properties of time-delay systems has been presented. The proposed approach relies on the introduction of the Extended Lie bracket operation which reduces to the standard Lie bracket for systems without delays. This operation has allowed to define integrability conditions for modules associated to systems with delays. As an example the linear equivalence problem for time-delay systems was addressed and solved.

\section{APPENDIX}

Proof of Theorem 1: Let $\omega_{i}(\mathbf{x}, \delta)=\sum_{k=0}^{\bar{s}} \omega_{i}^{k}(\mathbf{x}) \delta^{k}$ be the $i$-th row of the matrix $T^{-1}(\mathbf{x}, \delta)$. Setting $\gamma=s+\bar{s}$, by construction $\forall \alpha, l \in[1, n], \omega_{\alpha}(\mathbf{x}, \delta) r_{l}(\mathbf{x}, \delta)=\sum_{i=0}^{\gamma} Q_{\alpha l}^{i} \delta^{i}$, that is, for $i=$ $0 \cdots \gamma, \sum_{j=0}^{i} \omega_{\alpha}^{j}(\mathbf{x}) r_{l}^{i-j}(\mathbf{x}(-j))=Q_{\alpha l}^{i}$. We thus have $\forall p \geq 0$, $i \in[0, \gamma]$

$$
\frac{\partial}{\partial x(t-p)}\left(\sum_{j=0}^{i} \omega_{\alpha}^{j}(\mathbf{x}) r_{l}^{i-j}(\mathbf{x}(-j))\right)=0 .
$$

Equation (18) immediately implies

$$
\begin{aligned}
\sum_{p=0}^{q} \frac{\partial}{x(t-p)}\left(\sum_{\eta=0}^{k} \omega_{\alpha}^{\eta}(\mathbf{x}) r_{l}^{k-\eta}(\mathbf{x}(-\eta))\right) \\
-\sum_{p=0}^{k} \frac{\partial}{\partial x(t-p)}\left(\sum_{\eta=0}^{q} \omega_{\alpha}^{\eta}(\mathbf{x}) r_{j}^{q-\eta}(\mathbf{x}(-\eta))\right)=0
\end{aligned}
$$

Assuming without loss of generality $q \leq k$ and setting $\Omega_{i p}^{\alpha}=$ $\left(\partial\left(\omega_{\alpha}^{i}(\mathbf{x})\right)^{T} / \partial x(t-p)\right)-\left(\partial\left(\omega_{\alpha}^{p}(\mathbf{x})\right)^{T} / \partial x(t-i)\right)^{T},(19)$ leads to

$$
\begin{aligned}
\sum_{p=0}^{q} \sum_{i=0}^{k}\left(r_{l}^{k-i}(\mathbf{x}(-i))\right)^{T} \Omega_{i p}^{\alpha} r_{j}^{q-p}(\mathbf{x}(-p)) \\
+\left(\omega_{\alpha}^{0}(\mathbf{x}) \cdots \omega_{\alpha}^{q}(\mathbf{x})\right)\left[r_{j}^{q}, r_{l}^{k}\right]_{E q}=0 .
\end{aligned}
$$

Necessity: Assume now that $\omega_{\alpha}(\mathbf{x}, \delta) d x=\sum_{i=0}^{\bar{s}} \omega_{\alpha}^{i}(\mathbf{x}) \delta^{i} d x$ is exact, then for any $i, p \in[0, \gamma], \Omega_{i p}^{\alpha}=0$. Assuming without loss of generality $q \leq k$, from (20) we get that necessarily

$$
\left(\begin{array}{ccc}
\omega^{0}(\mathbf{x}) & \cdots & \omega^{q}(\mathbf{x}) \\
0 & \ddots & \vdots \\
0 & 0 & \omega^{0}(\mathbf{x}(-q))
\end{array}\right)\left[r_{j}^{q}, r_{l}^{k}\right]_{E q}=0
$$

which due to the full rank of $\omega^{0}(\mathbf{x})$, proves (12).

Sufficiency: By assumption $P_{n}(\mathbf{x}, \delta)=T(\mathbf{x}, \delta) Q(\delta)=$ $\sum_{j=0}^{s} P_{n j} \delta^{j}$, that is $P_{n k}=\sum_{i=0}^{k} T^{i}(\mathbf{x}) Q_{k-i}(-i)=$ $\sum_{i=0}^{k} T^{i}(\mathbf{x}) Q_{k-i}$.

Let $\operatorname{adj}[Q(\delta)]=\sum_{i=0}^{\gamma} \mathcal{S}_{i} \delta^{i}$ be the adjugate matrix associated to $Q(\delta)=\sum_{i=0}^{\gamma} Q_{i} \delta^{i}$. Then, since $Q(\delta) \operatorname{adj}[Q(\delta)]=[I d] \operatorname{det}(Q(\delta))=$ $[I d]\left(q_{0}+q_{1} \delta+\cdots+q_{t} \delta^{t}\right) \delta^{\beta}$, we have that $\sum_{i=0}^{l} Q_{i} \mathcal{S}_{l-i}=0$ for $l \leq \beta-1$ while $\sum_{i=0}^{\beta} Q_{i} \mathcal{S}_{\beta-i}=[I d] q_{0} \neq 0$. It follows that $\sum_{i=0}^{l} P_{n i}(-j) \mathcal{S}_{l-i}=0$ for $l \leq \beta-1$ and $\sum_{i=0}^{\beta} P_{n i}(-j) \mathcal{S}_{\beta-i}=$ $T^{0}(-j) q_{0}$

Consider now (20). Since condition (12) is satisfied, for any couple of indices $k, q$

$$
\sum_{p=0}^{q} \sum_{i=0}^{k}\left(r_{l}^{k-i}(\mathbf{x}(-i))^{T} \Omega_{i p}^{\alpha} r_{j}^{q-p}(\mathbf{x}(-p))=0\right.
$$

that is, $\Gamma_{k}^{q}(l)=\sum_{p=0}^{q} \sum_{i=0}^{k}\left(r_{l}^{k-i}(\mathbf{x}(-i))\right)^{T} \Omega_{i p}^{\alpha} P_{n, q-p}(\mathbf{x}(-p))=$ 0 . It follows that:

$$
\sum_{q=0}^{\beta} \Gamma_{k}^{q}(l) \mathcal{S}_{\beta-q}=\sum_{i=0}^{k}\left(r_{l}^{k-i}(\mathbf{x}(-i))\right)^{T} \Omega_{i 0}^{\alpha} T^{0} q_{0}=0
$$

which due to the invertibility of $T^{0} q_{0}$, implies that

$$
\tilde{\Gamma}_{k}(l)=\sum_{i=0}^{k}\left(r_{l}^{k-i}(\mathbf{x}(-i))\right)^{T} \Omega_{i 0}^{\alpha}=0 .
$$

For $l \in[1, n]$, and $k \in[0, \beta]$ we get $\tilde{\Gamma}_{k}=$ $\sum_{j=0}^{k}\left[P_{n, k-j}(\mathbf{x}(-j))\right]^{T} \Omega_{j 0}^{\alpha}=0$. Then $\sum_{i=0}^{\beta} \mathcal{S}_{\beta-i}^{T} \tilde{\Gamma}_{i}=$ $q_{0}\left[T^{0}\right]^{T} \Omega_{00}^{\alpha}=0$ that is $\Omega_{00}^{\alpha}=0$. 
By induction, assume that $\Omega_{i p}=0 \forall i \geq 0$ when $p \in[0, \bar{q}-1]$ and for $i \in[0, \bar{k}-1]$ when $p=\bar{q}$, then $\forall \gamma \geq 0$

$$
\begin{aligned}
0 & =\sum_{t=0}^{\beta} \Gamma_{\bar{k}+\gamma}^{\bar{q}+t}(l) \mathcal{S}_{\beta-t} \\
& =\sum_{i=0}^{\bar{k}+\gamma}\left[r_{l}^{\bar{k}+\gamma-i}(\mathbf{x}(-i))\right]^{T} \Omega_{i \bar{q}}^{\alpha} T^{0}(\mathbf{x}(-\bar{q})) q_{0}
\end{aligned}
$$

that is, due to the invertibility of $T^{0}(\mathbf{x}(-\bar{q})) q_{0}, \quad \sum_{i=0}^{\bar{k}+\gamma}$ $\left[r_{l}^{\bar{k}+\gamma-i}(\mathbf{x}(-i))\right]^{T} \Omega_{i \bar{q}}^{\alpha}=0$ or equivalently $\sum_{i=0}^{\gamma}$ $\left[r_{l}^{\gamma-i}(\mathbf{x}(-\bar{k}-i))\right]^{T} \Omega_{\bar{k}+i, \bar{q}}^{\alpha}=0$. One thus gets $0=\sum_{\gamma=0}^{\beta} \sum_{i=0}^{\gamma}$ $\mathcal{S}_{\beta-\gamma}^{T}\left[P_{n, \gamma-i}(\mathbf{x}(-\bar{k}-i))\right]^{T} \Omega_{\bar{k}+i, \bar{q}}^{\alpha}$ that is $0=q_{0}\left[T^{0}(\mathbf{x}(-\bar{k}))\right]^{T} \Omega_{\bar{k}, \bar{q}}^{\alpha}$ which proves the thesis, $q_{0}\left[T^{0}(\mathbf{x}(-\bar{k}))\right]^{T}$ being invertible.

\section{REFERENCES}

[1] P. S. Banks, "Nonlinear delay systems, Lie algebras and Lyapunov transformations," J. Math. Cont. Inf., vol. 19, pp. 59-72, 2002.

[2] N. Bekiaris-Liberis and M. Krstic, "Delay-adaptive feedback for linear feedforward systems," Syst. Control Lett., vol. 59, pp. 277-283, 2010.

[3] C. Califano, S. Monaco, and D. Normand-Cyrot, "On the problem of feedback linearization," Syst. Control Lett., vol. 36, pp. 61-67, 1999.

[4] C. Califano, L. A. Marquez-Martinez, and C. H. Moog, "On linear equivalence for time delay systems," in Proc. Amer. Control Conf., Baltimore, MD, 2010, pp. 6567-6572.

[5] A. Germani, M. Manes, and P. Pepe, "Input-output linearization with delay cancellation for nonlinear delay systems: The problem of the internal stability," Int. J. Rob. Nonlin. Contr., vol. 13, pp. 909-937, 2003.

[6] H. Gluesing-Luerssen, Linear Delay-Differential Systems with Commensurate Delay: An Algebraic Approach, ser. Lecture Notes in Mathematics. Berlin, Germany: Springer-Verlag, 2002, vol. 1770.

[7] H. Gorecki, S. Fuska, P. Grabowski, and A. Korytowski, Analysis and Synthesis of Time-Delay Systems. Warsaw, Poland: Wiley, 1989.

[8] K. Gu, V. L. Kharitonov, and J. Chen, Stability of Time Delay Systems. Boston, MA: Birkhäuser, 2003.

[9] A. Isidori, Nonlinear Control Systems: An Introduction, 3rd ed. London, U.K.: Springer-Verlag, 1995.

[10] A. Isidori, A. J. Krener, C. G. Giorgi, and S. Monaco, "Nonlinear decoupling via feedback: A differential geometric approach," IEEE Trans. Autom. Control, vol. AC-26, no. 2, pp. 331-345, Apr. 1981.

[11] B. Jakubczyk and W. Respondek, "On linearization of control systems," Bull. Acad. Polonaise Sci., vol. 28, pp. 517-522, 1980.

[12] V. Kolmanovskii and A. Myshkis, Applied Theory of Functional Differential Equations. Dordrecht, The Netherlands: Kluwer, 1992, ch. 1.

[13] A. J. Krener, "On the equivalence of control systems and the linearization of nonlinear systems," SIAM J. Contr., vol. 11, pp. 670-676, 1973.

[14] M. Krstic, Delay Compensation for Nonlinear, Adaptive, and PDE Systems. Boston, MA: Birkhäuser, 2009.

[15] S. Ibrir, "Observer-based control of a class of time-delay nonlinear systems having triangular structure," Automatica, vol. 47, pp. 388-394, 2011.

[16] H. G. Lee, A. Arapostathis, and S. I. Marcus, "On the linearization of discrete-time systems," Int. J. Control, vol. 45, pp. 1783-1785, 1987.

[17] L. A. Marquez-Martinez and C. H. Moog, "New insights on the analysis of nonlinear time-delay systems: Application to the triangular equivalence," Syst. Control Lett., vol. 56, pp. 133-140, 2007.

[18] L. A. Marquez-Martinez, C. H. Moog, and M. Velasco-Villa, "Observability and observers for nonlinear systems with time delay," Kybernetika, vol. 38, no. 4, pp. 445-456, 2002.

[19] W. Michiels and S.-I. Niculescu, Stability and Stabilization of TimeDelay Systems. An Eigenvalue-Based Approach. Philadelphia, PA: SIAM, 2007, vol. 12, Advances in Design and Control.

[20] S. Monaco and D. Normand-Cyrot, "On the immersion under feedback of a multidimensional discrete-time nonlinear system into a linear system," Int. J. Control, vol. 38, pp. 245-261, 1983.

[21] S. Monaco and D. Normand-Cyrot, "A unifying representation for nonlinear discrete-time and sampled dynamics," J. Math. Syst. Est. Contr., vol. 5, pp. 103-105, 1995, V.7, (1997), pp. 477-503.

[22] S.-I. Niculescu, Delay Effects on Stability. London, U.K.: SpringerVerlag, 2001.
[23] T. Oguchi, "A finite spectrum assignment for retarded non-linear systems and its solvability condition," Int. J. Control, vol. 80, pp. 898-907, 2007.

[24] T. Oguchi, A. Watanabe, and T. Nakamizo, "Input-output linearization of retarded non-linear systems by using an extension of Lie derivative," Int. J. Control, vol. 75, no. 8, pp. 582-590, 2002.

[25] J. F. Pommaret and A. Quadrat, "Generalized Bezout identity," Applicable Algebra Eng., Comm. Comp., vol. 9, pp. 91-116, 1998.

[26] J. P. Richard, "Time-delay systems: An overview of some recent advances and open problems," Automatica, vol. 39, pp. 1667-1694, 2003.

[27] W. M. Wonham and A. S. Morse, "Decoupling and pole assignment in linear multivariable systems: A geometric approach," SIAM J. Contr., vol. 8 , pp. $1-18,1970$.

[28] X. Xia, L. A. Marquez-Martinez, P. Zagalak, and C. H. Moog, "Analysis of nonlinear time-delay systems using modules over non-commutative rings," Automatica, vol. 38, pp. 1549-1555, 2002. 